\title{
Working Group 6 Summary: Spin and 3D Structure
}

\author{
Oleg Eyser \\ Brookhaven National Laboratory \\ E-mail: keyserabnl.gov

\section{Bakur Parsamyan} \\ CERN, INFN and University of Turin \\ E-mail: bakurecern.ch

\section{Ted Rogers} \\ Old Dominion University and Jefferson Lab \\ E-mail: trogers@odu.edu
}

The spin and 3D structure session of the DIS2019 conference focused on recent efforts to understand nucleon structure using collinear factorization theorems, transverse momentum dependent correlation functions (TMDs), generalized parton distribution (GPDs) and similar objects. A large amount of progress in both theoretical and experimental directions was reported. We summarize some of the highlights here.

XXVII International Workshop on Deep-Inelastic Scattering and Related Subjects - DIS2019 8-12 April, 2019

Torino, Italy 


\section{Introduction}

In many applications of perturbative QCD at high energies, it is only the component of momentum in the direction of motion of the energetic hadrons that is important. The correlation functions needed for such calculations, the collinear parton densities, depend only on longitudinal momentum fractions. However, when the focus is hadron structure, more complicated objects are needed. These include the transverse momentum dependent (TMD) parton densities and the generalized parton densities (GPDs). The former is closely related to the probability to find intrinsic transverse momentum in a cross section, while the latter is related to the probability to find certain size configurations in an amplitude. Knowledge of such objects provide complimentary information about hadron and nucleon structure.

The spin and 3D structure efforts were primarily focused on measuring and calculating TMDs, GPDs, and similar types of objects that go beyond collinear and unpolarized factorization theory. Below we will start by describing experimental results and end with a summary of mainly theoretical progress, with sections focused on collinear functions, TMDs and GPDs.

\section{Hadron multiplicities and fragmentation functions related measurements}

In perturbative quantum chromodynamics ( $\mathrm{pQCD}$ ), quark fragmentation into hadrons is described by non-perturbative fragmentation functions (FFs). Studying the production of different hadron species in SIDIS is one of the main tools to explore the fragmentaion process. On the one hand, this approach gives a possibility to study $q$ and $\bar{q}$ fragmentation separately making full flavour separation. On the other hand, in the pQCD description of SIDIS process, FFs can be accessed only in form of convolutions with parton distribution functions (PDFs). Perturbative QCD theory cannot directly predict values of multiplicities. It does, however, provide estimates of the limits of multiplicity ratios. Recently COMPASS published results for $K^{-} / K^{+}$multiplicity ratio, $R_{K}$, from isoscalar (deuteron) target data for kaons produced in DIS in the region of high fraction $z$ of the virtual-photon energy [1]. The results were found to be up to a factor of 2.5 below the lower limit obtained from LO and NLO pQCD calculations. In addition, a strong dependence on the fraction of the incoming moon energy carried by the virtual photon, $v$, has been observed, which is also not expected in $\mathrm{pQCD}$. It was demonstrated that both these phenomena could be described by the missing mass variable, suggesting that within the pQCD formalism an additional correction may be required to account for the limitations on the phase space available for hadronization.

COMPASS has presented further progress on deuteron data analysis including results obtained for $\bar{p} / p$ multiplicity ratio, $R_{p}$, for protons produced at large $z$ and new results for $R_{K}$ in an extended kinematic phase-space [2]. In particular it was shown that, for $R_{p}$ the discrepancy with respect to the LO QCD expectations is much larger compared to the kaon case (probably due to even stronger phase space limitations) and that, similar to $R_{K}$, proton multiplicity ratio also exhibits similar missing mass dependence (see Fig. 1, left panels). Updated kaon results demonstrate that $R_{K}$ tends to saturate at high values of $v$ approaching the pQCD expectations (see Fig. 1, right panel). Similar trend was observed also for $R_{p}$.

These observations suggest that discussed phenomena may have a stronger impact on the measurements obtained by the experiments operating at lower center-of-mass energies and that the 

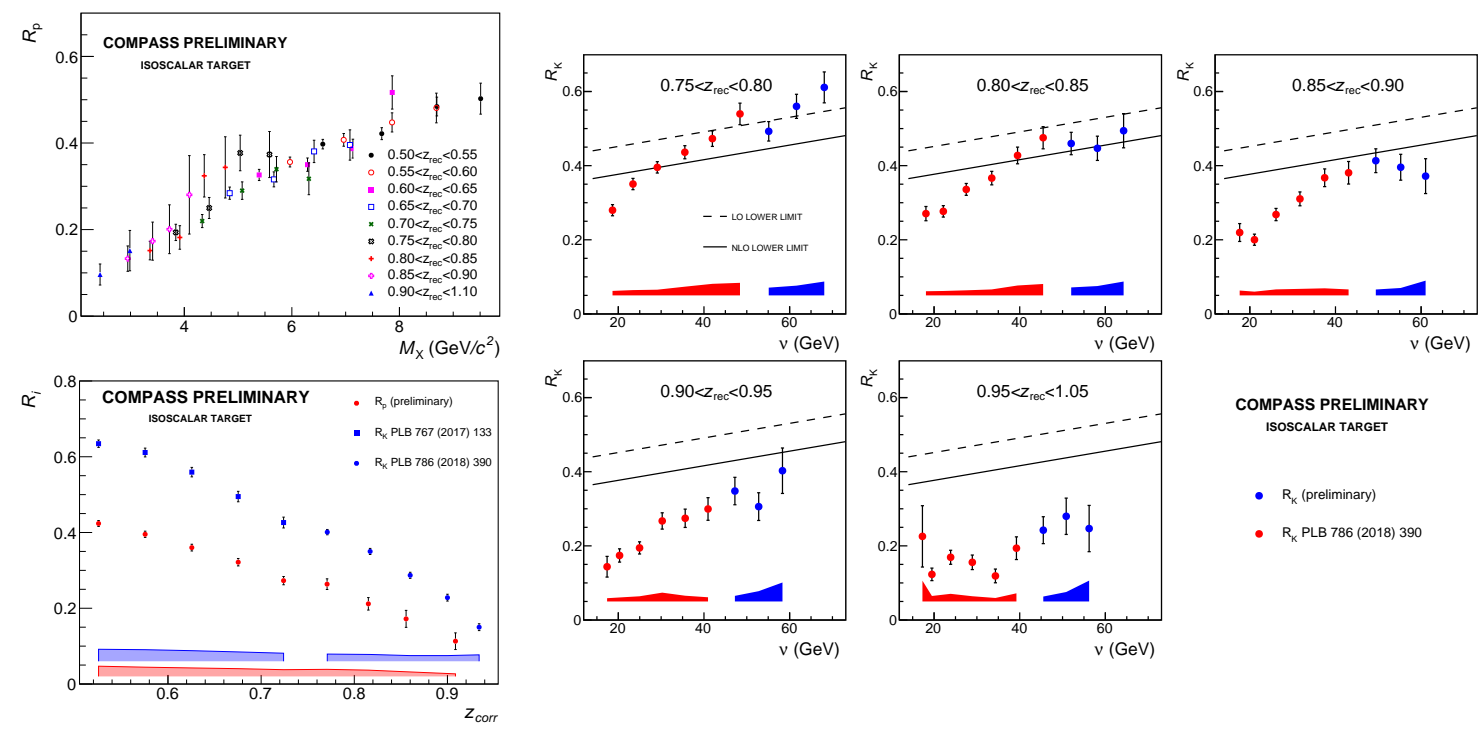

- $\mathrm{R}_{\mathrm{K}}$ PLB $786(2018) 390$

Figure 1: COMPASS results for $R_{p}$ and $R_{K}$. Left panel, top: The $R_{p}$ ratio as a function of the missing mass. Left panel, bottom: Comparison of $R_{p}$ and $R_{K}$. Right panel: preliminary results on $R_{K}$ measured in an extended $v$ range compared to Ref. [1].

region of applicability of factorised PQCD in SIDIS should be generally revised.

In addition to multiplicities obtained from deuteron data, COMPASS has presented preliminary results for kaon multiplicities obtained from a fraction of data collected with a proton (liquid hydrogen) target [3]. The multiplicities $M^{K^{ \pm}}$were extracted using three-dimensional representation in Bjorken $x$, the fraction of the beam lepton energy carried by the virtual photon $y$, and $z$ variables. The results have been corrected for QED radiative effects and diffractive vector meson contamination. In addition to $M^{K^{ \pm}}$ also kaon multiplicity sums and ratios have been presented in comparison with COMPASS deuteron results. Kaon multiplicity sums on proton were found to be compatible with the deuteron ones, while $R_{K}$ s from proton data are significantly higher compared to the deuteron case. Both observations are in agreement with $\mathrm{pQCD}$ expectations. Preliminary COMPASS results were also compared to the HERMES data [4] (see Fig. 2). A known discrepancy between COMPASS and

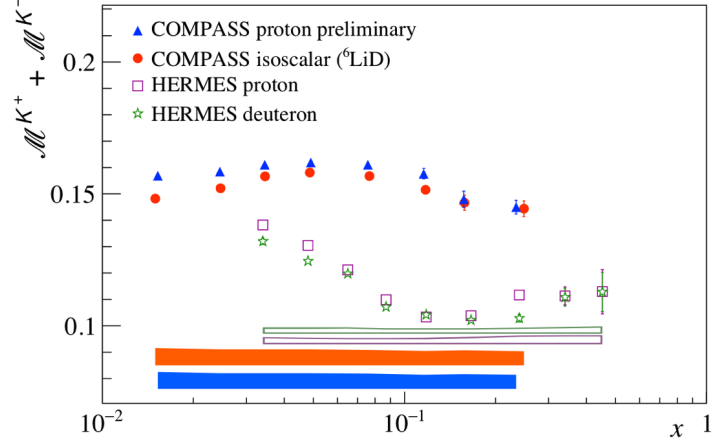

Figure 2: Multiplicity sums for kaons from COMPASS (preliminary) and HERMES.

HERMES deuteron data (taken at much different beam energies) persists also for the proton case.

Apart from kaon multiplicities COMPASS has presented also preliminary proton data results for hadron transverse momentum, $P_{h T}$ dependent hadron multiplicities, evaluated over threedimensional grid in $x$, photon virtuality $Q^{2}$ and $z$ [5]. Measurement of $P_{h T}$-multiplicities provides 
an important information about intrinsic transverse motion of the partons inside the nucleon parameterized by Transverse Momentum Dependent (TMD) PDFs and about the role of TMD effects in the fragmentation process (TMD FFs).

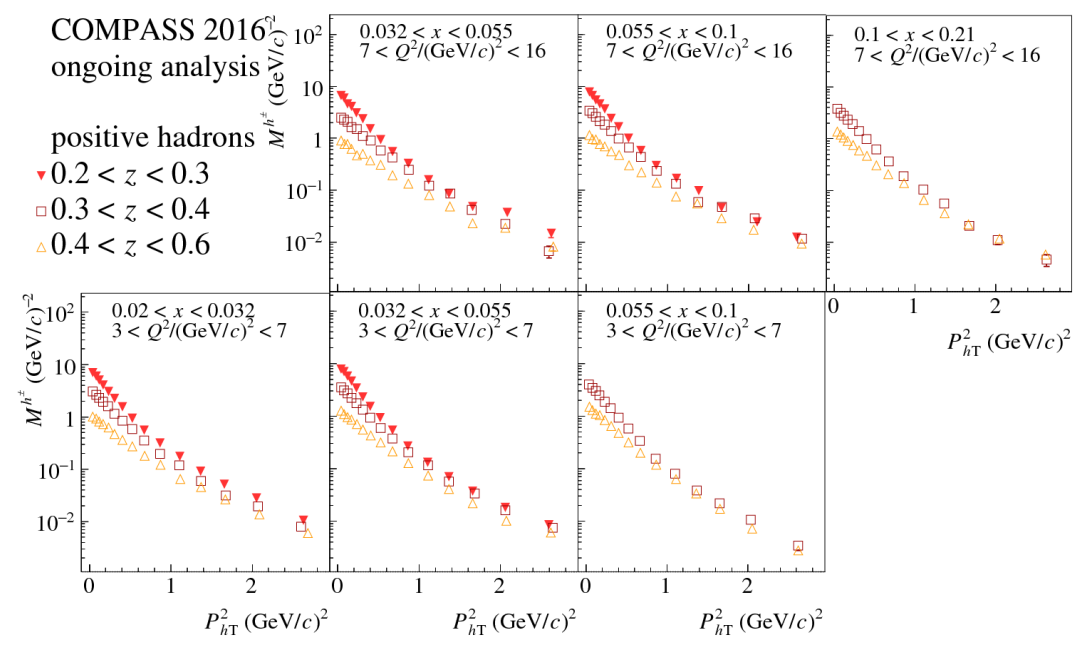

Figure 3: COMPASS positively-charged hadron $P_{h T}$-dependent multiplicities.

Presented preliminary results (see Fig. 3) were extracted from a small fraction of collected proton data and for restricted kinematic domain where radiative effects and diffractive vector meson contamination contributions were estimated to be negligible. The results are in agreement with previous COMPASS measurements done with isoscalar target.

Belle collaboration has presented the first direct transverse-momentum-dependent single-hadron production cross sections in $e^{+} e^{-}$collisions for pions, kaons, and protons [6, 7]. These results are directly related to the unpolarized single-hadron fragmentation functions and their transverse momentum dependence. The cross sections were measured at $\sqrt{s}=10.58 \mathrm{GeV}$ as a function of fractional energy $z$, the event-shape variable called thrust ${ }^{1}$, and the transverse momentum with respect to the reference (thrust) axis (see Fig. 4). It has been shown that a Gaussian functional form describes well the transverse-momentum dependence at small transverse momenta. The Gaussian widths were found to vary with $z$ and thrust. This long desired input provided by the Belle experiment is important to obtain a better theoretical description of the various transverse-momentumdependent and related higher-twist effects related to spin-dependent asymmetries in semi-inclusive deep inelastic scattering, proton-proton collisions and $e^{+} e^{-}$annihilation. In addition, these results provide the unpolarized baseline for any polarized, transverse-momentum-dependent fragmentation functions such as the Collins FF. In parallel, the experiment continues working on dihadron transverse momentum dependent fragmentation studies and e.g multi-dimensional analysis of Collins asymmetries for pion and kaon combinations [6].

Further interesting measurements related to study of FFs and dihadron FFs via extraction of various weighted asymmetries at Belle, CLAS12 and future EIC have been suggested during the conference $[8,9]$.

\footnotetext{
${ }^{1}$ the variable describes how collimated are the particles in the event
} 


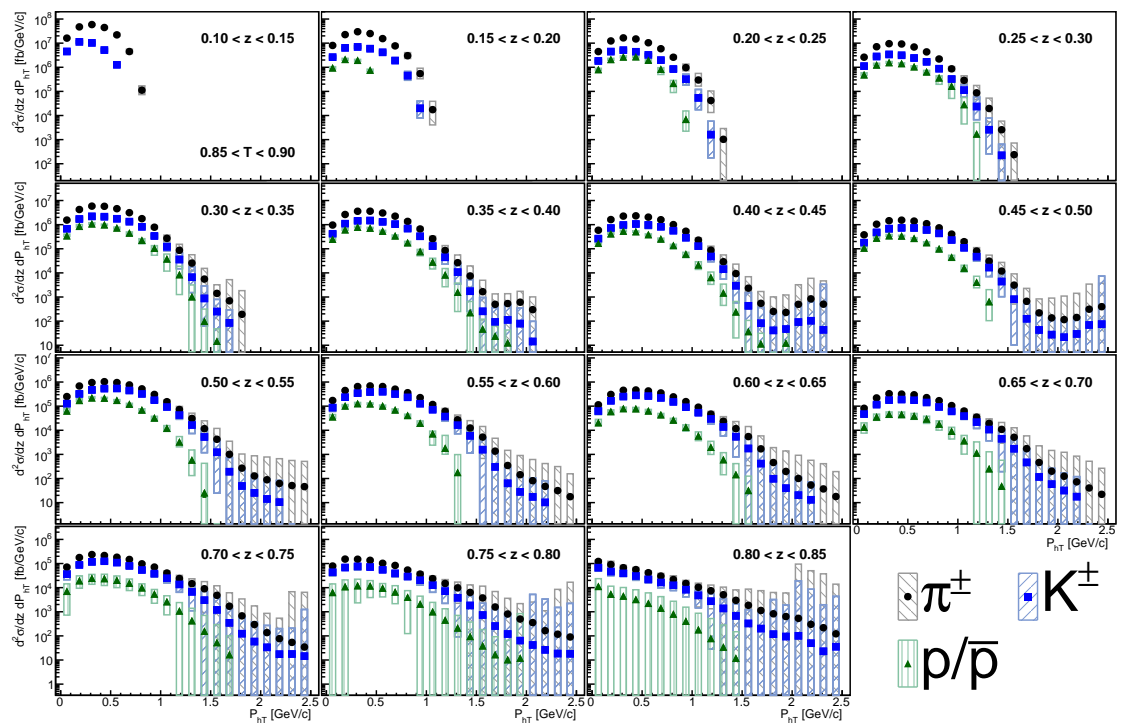

Figure 4: Belle results for differential cross sections for pions, kaons and protons as a function of $P_{h T}$ for the indicated $\mathrm{z}$ bins and thrust $0.85<\mathrm{T}<0.9$

\section{SIDIS and Drell-Yan measurements of azimuth and target polarization (in)dependent asymmetries}

Measurement of azimuth and target polarization (in)dependent asymmetries in SIDIS and Drell-Yan is an important source of information to access TMD PDFs. The TMD PDFs are universal, process-independent functions ${ }^{2}$ [10] describing longitudinal and transverse momenta distributions of partons and their correlations with nucleon and quark spins. Within Leading Order (LO) QCD parton model approach the polarized nucleon is described by six time reversal even and two time reversal odd twist-2 quark TMD PDFs. Correlations between nucleon and quark spins and quark intrinsic momenta induce azimuthal modulations (asymmetries) in the cross sections of SIDIS and of Drell-Yan (DY) process. Applying the TMD factorization theorems [10] allows one to express the asymmetries arising in DY and SIDIS cross sections in terms of convolutions of perturbatively calculable hard-scattering parton cross sections, hard-scale dependent TMD PDFs and (for SIDIS) parton fragmentation functions.

Several new results on azimuth and target spin (in)dependent asymmetries from HERMES, COMPASS and CLAS12 collaborations were presented. Starting from polarization independent asymmetries, COMPASS experiment has shown preliminary proton results for all three SIDIS amplitudes [11]: $A_{U U}^{\cos \left(\phi_{h}\right)}$ (Cahn effect), $A_{U U}^{\cos \left(2 \phi_{h}\right)}$ (Boer-Mulders effect) and $A_{L U}^{\sin \left(\phi_{h}\right)}$ (beam-spin, or beam-helicity asymmetry). The first observable is a sub-leading $\left(Q^{-1}\right.$-suppressed) or higher-twist asymmetry, which was first predicted as a kinematic effect that would appear in presence of nonzero intrinsic transverse momentum, $\mathbf{k}_{\mathbf{T}}$, of the quarks inside the nucleon. The $A_{U U}^{\cos \left(2 \phi_{h}\right)}$ leadingtwist amplitude is related to the correlation between the quark spin and $\mathbf{k}_{\mathbf{T}}$, which is parameterized by the Boer-Mulders TMD PDFs. Last unpolarized term, the $A_{L U}^{\sin \left(\phi_{h}\right)}$ asymmetry, is again a highertwist observable related to four twist-2/twist-3 mixed combinations of different PDFs and FFs.

\footnotetext{
${ }^{2} \mathrm{QCD}$ generalized universality: time-reversal modified process-independence of TMD PDFs
} 
Presented COMPASS results for Cahn and Boer-Mulders asymmetries exhibit strong kinematic dependences as a function of $x, z$ and $P_{h T}$ confirming the results of previous COMPASS measurements with isoscalar target [12], while for $A_{L U}^{\sin \left(\phi_{h}\right)}$ no significant signal is observed (see Fig. 5).

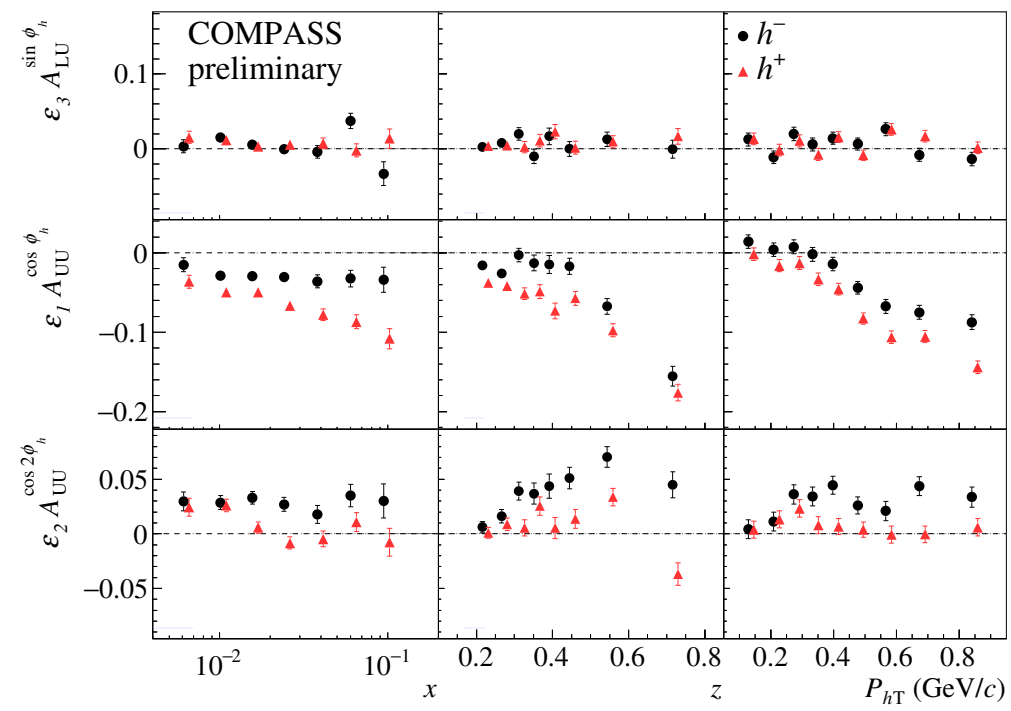

Figure 5: COMPASS azimuthal asymmetries for charged hadrons produced in SIDIS off a proton target.

The collaboration also studied the contribution to the unpolarized SIDIS asymmetries coming from diffractively produced vector mesons. It was shown that in certain kinematic regions the impact can be significant, explaining in part the kinematic dependencies and helping the interpretation of the measurements.

HERMES collaboration presented new results on beam-helicity asymmetries for pions, kaons, protons and antiprotons from hydrogen and deuterium SIDIS data [13, 14]. Significant non-zero signal has been observed for charged pions and positively charged kaons, while consistent with zero for other hadrons. Shown in Fig. 6 (left panel) comparison of HERMES and CLAS proton results is done after scaling them by $Q$ in order to compensate the $Q^{-1}$ suppression inherent to subleading asymmetries. While both experiments see significant amplitudes, the results diverge in certain kinematic ranges, which can be attributed to the complex nature of the asymmetry and kinematicsdependent dominance or suppression of its different ingredients. First preliminary results on $A_{L U}^{\sin \left(\phi_{h}\right)}$ shown by CLAS12 [15] reconfirm the non-zero effect (see Fig. 6 (right panel)). The results were based on a small fraction of collected data. In addition to the one-dimensional analysis, HERMES has carried out also the three-dimensional $\left(x, z\right.$, and $\left.P_{h T}\right)$ extraction of the beam-helicity asymmetry. Similar multi-differential approach is planned to be applied in near future both by COMPASS and CLAS12 collaborations.

Apart from the target spin independent asymmetries, HERMES has presented a reanalysis of the complete SIDIS sample collected by the experiment with longitudinally polarized protons and deuterons. The analysis was focused on the two double longitudinal spin dependent asymmetries (DLSAs) related to the azimuth-independent, $F_{L L}^{h}$, and the cosine-modulated, $F_{L L}^{h, \cos (\phi)}$, structure functions $[16,17]$. In the collinear limit, $F_{L L}^{h}$ reduces to the well-known product of the collinear 

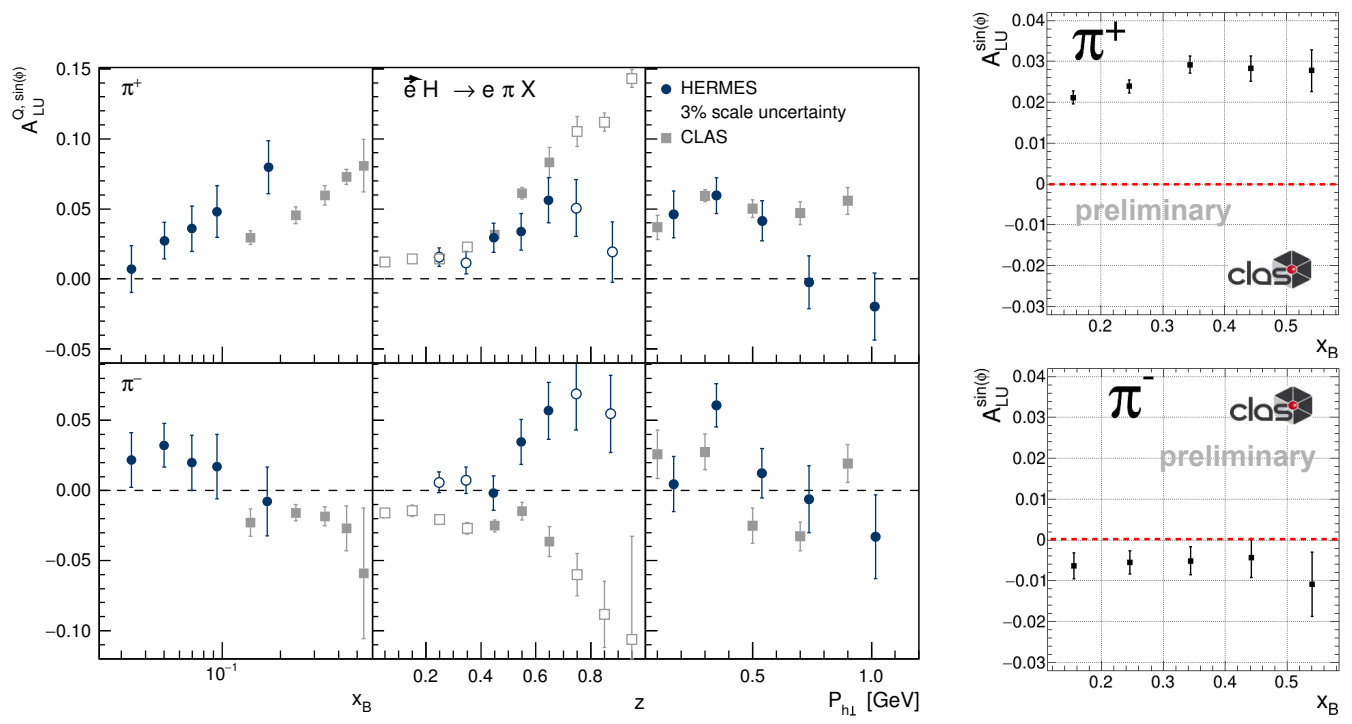

Figure 6: Left panel: HERMES and CLAS proton beam-spin asymmetry ( $Q$-scaled) for charged pions produced in SIDIS off a proton target. Right panel: preliminary results for beam-spin asymmetry from CLAS12.

helicity distribution $g_{1}^{q}(x)$ and the ordinary collinear fragmentation function $D_{1, q}^{h}$. The $F_{L L}^{h, \cos (\phi)}$ is a subleading effect, which in Wandzura-Wilczek-type approximation is also related to the convolution of helicity PDF and ordinary FF [18, 19, 20]. HERMES performed first ever extraction of the three-dimensional dependence of the DLSAs. The azimuthal DLSA is found to be compatible with zero within the uncertainties, which might be explained by the subleading suppression of the effect. At the same time, the azimuth-independent double spin asymmetry showed an expectedly non-vanishing effect. Within the precision of the measurements the asymmetries, however, do not exhibit any obvious dependence on the hadron variables (see Fig. 7). Presented by HERMES unique multidimensional data are expected to provide an essentially model-independent constraint for theory and phenomenological analyses.

Apart from the unpolarized and helicity PDFs certain attention has been driven to the transversity PDF and related measurements and theoretical developments [21, 22, 23]. An alternative way to extract the transversity PDF building the so-called difference asymmetries based on available data on Collins asymmetries alone, has been presented [21, 24]. This method, in particular, allows extracting combinations of the $u$ and $d$ quark transversity PDF without requiring specific knowledge of the Collins FF. This is at variance with standard approaches, which necessarily rely on available parameterizations of Collins FF extracted e.g. from $e^{+} e^{-}$data. Ratios of the $u$ and $d$ quark transversity distributions have been extracted from the COMPASS measurements of the Collins asymmetries of positively and negatively charged hadrons produced on transversely polarized proton and deuteron targets.

As shown in Fig. 8 (left panel), the results for the ratio $h_{1}^{u_{v}} / h_{1}^{d_{v}}$ compare well to those obtained with standard approaches. Due to a lack of precise Collins asymmetry measurements with isoscalar targets, the statistical precision of $d$-quark transversity is poor [21, 22]. In order to improve this situation, COMPASS is planning to dedicate a full year of data taking in 2021 to the measurements 


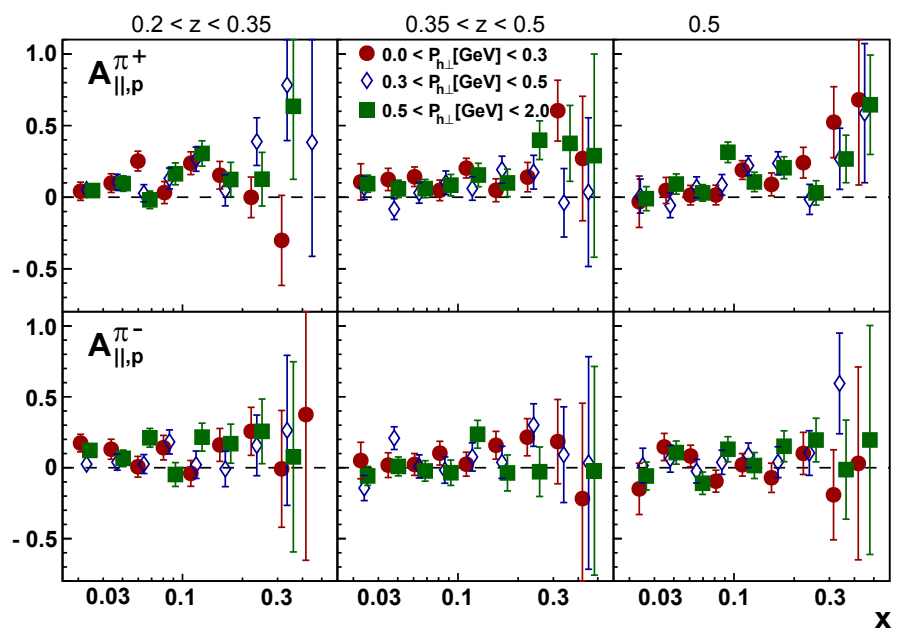

Figure 7: HERMES longitudinal double spin asymmetry as a function of $x$ in three different $z$ ranges and three different $P_{h T}$ ranges.
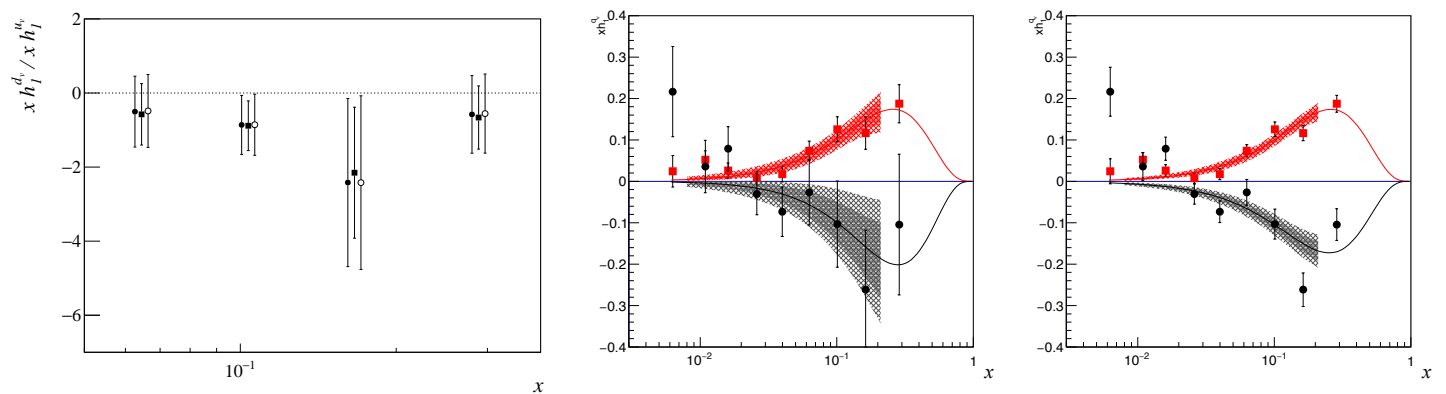

Figure 8: Left panel: Ratio $h_{1}^{u_{v}} / h_{1}^{d_{v}}$ evaluated using COMPASS difference asymmetries (closed circles and squares) compared to the results of point-by-point extraction from Ref. [25]. Middle panel: the values of $x h_{1}^{u_{v}}$ (red squares) and $x h_{1}^{d_{v}}$ (black circles) obtained using existing proton and deuteron SIDIS data. Right panel: same as the left plot, but with the projected impact of future COMPASS deuteron data to be collected in 2021 (the bands correspond to 68\% and 90\% confidence intervals).

with transversely polarized deuteron target [23]. Projected improvements for both $u$ and $d$ quark transversity distributions are illustrated in Fig. 8 (middle and right panels).

Apart from azimuthal effects in single-hadron production also dihadron asymmetries have been discussed. In particular, CLAS12 collaboration has shown preliminary results for dihadron beam-spin and longitudinal spin dependent asymmetries [26]. These first results obtained with small fraction of projected CLAS12 data have been discussed in connection with available CLAS6 and COMPASS preliminary data [27]. Theoretical interpretation of longitudinal spin dependent dihadron asymmetries and predictions for COMPASS kinematics have been presented [28].

Complementary to SIDIS measurements, COMPASS reported on the progress obtained for Drell-Yan measurements with transversely polarized proton target. New data sample collected in 2018 has been partially analysed and obtained preliminary results for Drell-Yan transverse spin asymmetries (TSAs) have been shown in combination with published results from 2015 sam- 
ple $[29,30]$. One of the main goals of the measurement is the study of the Sivers function, which plays an important role among the TMD PDFs. In the TMD framework of QCD it is predicted that the two naively time-reversal odd TMD PDFs, i.e. the quark Sivers functions $f_{1 T}^{\perp}$ and BoerMulders functions $h_{1}^{\perp}$, have opposite sign when measured in SIDIS and in DY (or W/Z-boson) productions. This is considered as a fundamental test of the TMD-framework of QCD. The combined COMPASS result for the average Sivers asymmetry $A_{T}^{\sin \phi_{S}}$, obtained from 2015 data and analyzed fraction of 2018 data, is found to be above zero at about one standard deviation of the total uncertainty (see Fig. 9, left panel). The positive sign and the amplitude of the asymmetry is consistent with the predicted change of sign for the Sivers function between SIDIS and Drell-Yan measurements and is found to be compatible with various model predictions [31]. In addition to the studies carried out for canonical Drell-Yan TSAs, COMPASS has extracted also the so-called virtual photon transverse momentum, $q_{T}$-weighted TSAs from the same data-sample [32]. At variance to the TSAs, the $q_{T}$-weighted TSAs are interpreted not in terms of convolutions of TMD PDFs, but as products of $k_{T}^{2}$-moments of the TMD PDFs.
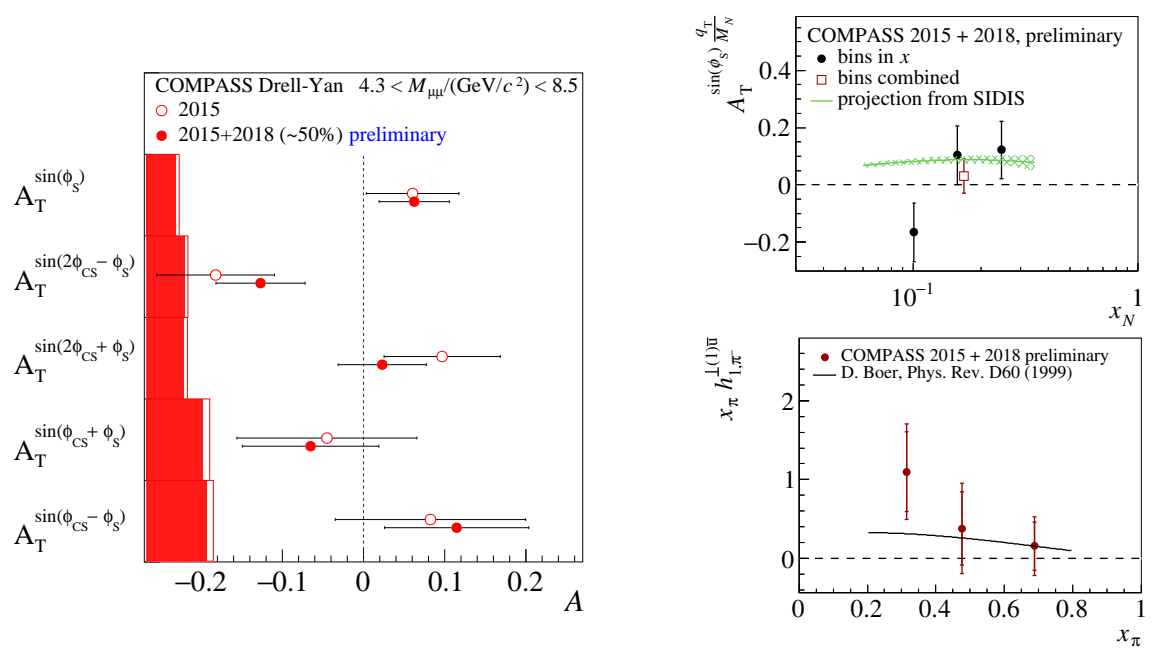

Figure 9: Left panel: COMPASS Drell-Yan TSAs integrated over the entire kinematic range. Right panel (top): The weighted Sivers asymmetry from COMPASS DY data, compared with the projection from SIDIS (assuming sign-change). Right panel (bottom): The Boer-Mulders function of the pion as extracted from COMPASS data compared with the parameterization from Ref. [33].

Such an approach simplifies the access to the PDFs and allows to make visual SIDIS-DY crossstudies. Two such examples are shown in Fig. 9 (right panel). The figure in the top panel shows a comparison of the $q_{T}$-weighted Drell-Yan asymmetry with the projection obtained using the first moment of the Sivers TMD PDF extracted from the measurement of weighted Sivers asymmetry in SIDIS. The second figure (right, bottom panel) shows the result obtained for the first moment of the pion Boer-Mulders function extracted from related Drell-Yan asymmetry using a parameterization for the transversity TMD PDF obtained from SIDIS data fits.

\section{Generalized Parton Distributions related measurements}

Measurements of exclusive single-photon and exclusive meson productions in hard lepton- 
nucleon scattering (Deeply Virtual Compton Scattering (DVCS) and Deeply Virtual Meson Production (DVMP) processes, correspondingly) provide important data for mapping of Generalised Parton Distributions (GPDs). COMPASS and CLAS12 experiments have reported about their recent progress for this kind of measurements $[34,35]$. Obtained by COMPASS preliminary exclusive single photon distributions are presented in Fig. 10. The analysis was carried out on the small fraction of data collected with liquid hydrogen target and is a continuation of analysis published in Ref. [36]. Similar to COMPASS, CLAS12 also presented preliminary results obtained only with a small fraction of projected data. Extracted by CLAS12 raw DVCS beam-spin asymmetry is presented in Fig. 10 (leftmost panel).
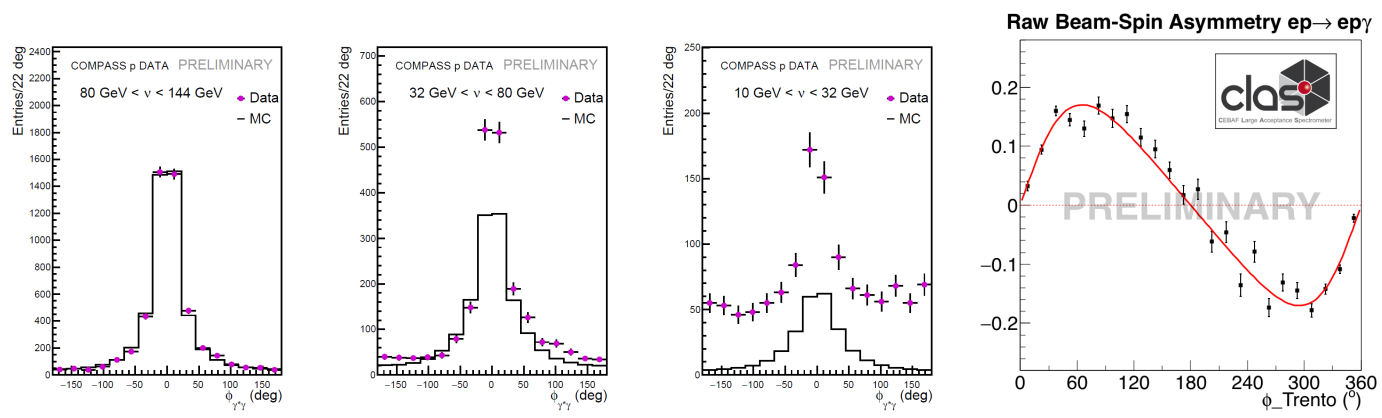

Figure 10: Right three panels: COMPASS preliminary exclusive single photon distributions are compared to the BH contribution normalized to the bin at small $v$ (visible $\pi^{0}$ contamination is subtracted but not yet invisible $\pi^{0}$ contamination). Leftmost panel: CLAS12 preliminary raw DVCS beam-spin asymmetry.

\section{Other Transverse Spin Phenomena}

Transverse spin asymmetries have a long history of revealing the richness of QCD from early fixed target experiments at center of mass energies of a few $\mathrm{GeV}$ up to proton-proton collisions at $500 \mathrm{GeV}$. Inclusive observables at midrapidity are typically consistent with zero [37], but they can still be used to constrain certain multiparton (gluon) correlators in a twist-3 framework. Recently, the PHENIX experiment showed transverse single spin asymmetries of charged hadrons at moderate forward rapidity in $p+p$ collisions at $200 \mathrm{GeV}$. In $p+\mathrm{Al}$ and $p+\mathrm{Al}$ collisions, a suppression of these asymmetries is observed that is consistent with $A^{\frac{1}{3}}$ scaling $[38,39]$. Such a suppression has been suggested as a test of gluon saturation in the CGC formalism in the past and it will be interesting to see what can be learned from this data.

In the last three sections, we will summarize theoretical work, organized according to the type of correlation functions that are the focus.

\section{Collinear Distributions}

There has been significant theoretical and phenomenological progress in the study of spindependent collinear parton distributions and spin asymmetries, including an updated analysis of the double spin asymmetries - see discussion of Figs. 6 and 7 above. RHIC experiments have 
shown results of double helicity asymmetries of inclusive and dijet production in proton-proton collisions at $510 \mathrm{GeV}$ [40]. These results include a full underlying event correction and they have since been published [41]. In conclusion of first part of the W-boson program, parity violating single spin asymmetries from PHENIX and STAR have been published and are available for the inclusion in global analyses to improve the determination of the sea quark polarization $[42,43$, 44]. New theoretical tools have been developed to extract $\Delta g$ in polarized semi-inclusive deep inelastic scattering using heavy quark production at NLO [45, 46], which will be important for the study of the gluon fusion process at a future EIC. M. Radici reported on updates of extractions of the proton tensor charge obtained from Mellin moments of the transversity distribution, which is important for high precision beyond-the-standard -model searches, and interesting tension with recent lattice calculations $[22,47,48]$ was described. Dihadron fragmentation functions (DiFFs), a type of collinear fragmentation function involving pairs of hadrons, have certain advantages such as allowing transversity to be probed in semiinclusive measurements of deep-inelastic lepton-nucleon scattering (SIDIS) without the need for TMD functions, and some new applications of DiFFs were discussed $[8,9]$.

\section{Generalized Parton Distributions}

Work was presented on progress in the study of GPDs, including the presentation of new global fits to deeply virtual Compton scattering (DVCS) in a leading order and leading twist formalism [49]. In addition, calculations within models [50, 51] addressing aspects needed for their implementation in lattice calculations and a study by A. Amor-Quiroz confirming the appearance of a difference between the Ji and Jaffe-Manohar spin decompositions at two-loop [52], while S. Rodini reported on a breakdown of the lensing relation in a model field theory when the final state involves many partons [53].

\section{Transverse Momentum Dependence}

Much progress was made in the last year on in the study of TMDs. Extensive progress on the global extraction of unpolarized TMDs, including evolution and high order calculations, was reported on [54]. The was also significant theoretical work on the Sivers function, including NLO calculations [55] and treatments of the non-perturbative evolution. S. Gómez discussed the possibility to study linearly polarized gluons in TMD factorization at NNLO.

In the large transverse momentum limit, the description in terms of TMDs transitions to one in terms of standard collinear factorization. Two talks discussed interesting tension that has been found with this matching in SIDIS [56, 57], and A. Simonelli discussed how knowledge of the transition to large transverse momentum is impacted by errors in collinear PDFs and FFs [58].

A promising avenue toward understanding TMDs is through an improved understanding of the hadronization process and its relation to processes in Monte Carlo event generators (MCEGs). Important work on this front was also reported on [59, 60]. 


\section{References}

[1] COMPASS collaboration, R. Akhunzyanov et al., $K$ over $K^{+}$multiplicity ratio for kaons produced in DIS with a large fraction of the virtual-photon energy, Phys. Lett. B786 (2018) 390-398, [1802.00584].

[2] COMPASS collaboration, M. Stolarski, High-z proton and kaon multiplicity ratios on deuteron target in SIDIS, PoS DIS2019 (2019) 207.

[3] COMPASS collaboration, N. Pierre, Kaon multiplicities in SIDIS from COMPASS, PoS DIS2019 (2019) 197.

[4] HERMES collaboration, A. Airapetian et al., Multiplicities of charged pions and kaons from semi-inclusive deep-inelastic scattering by the proton and the deuteron, Phys. Rev. D87 (2013) 074029, [1212.5407].

[5] COMPASS collaboration, A. Moretti, Transverse momentum dependent multiplicities of hadrons produced in DIS at COMPASS, 1907.07453.

[6] BELLE collaboration, R. Seidl et al., Transverse momentum dependent production cross sections of charged pions, kaons and protons produced in inclusive $e^{+} e^{-}$annihilation at $\sqrt{s}=10.58 \mathrm{GeV}$, Phys. Rev. D99 (2019) 112006, [1902 . 01552].

[7] BELLE collaboration, R. Seidl, Fragmentation related measurements at Belle, .

[8] A. Kotzinian, H. H. Matevosyan and A. W. Thomas, New way to access the quark fragmentation functions in electron-positron annihilation, 1907.08411.

[9] H. H. Matevosyan, A. Kotzinian and A. W. Thomas, Semi-inclusive back-to-back production of a hadron pair and a single hadron in $e^{+} e$ annihilation, JHEP 10 (2018) 008, [1808.00954].

[10] J. Collins, Foundations of perturbative QCD, Camb. Monogr. Part. Phys. Nucl. Phys. Cosmol. 32 (2011) 1-624.

[11] COMPASS collaboration, J. Matousek, Measurement of the azimuthal modulations of hadrons in unpolarised SIDIS, 1907.08851.

[12] COMPASS collaboration, C. Adolph et al., Measurement of azimuthal hadron asymmetries in semi-inclusive deep inelastic scattering off unpolarised nucleons, Nucl. Phys. B886 (2014) 1046-1077, [1401.6284].

[13] HERMES collaboration, G. Schnell, Beam-helicity asymmetries in semi-inclusive deep-inelastic single-hadron production from unpolarized hydrogen and deuterium targets, PoS DIS2019 (2019) 203.

[14] HERMES collaboration, A. Airapetian et al., Beam-helicity asymmetries for single-hadron production in semi-inclusive deep-inelastic scattering from unpolarized hydrogen and deuterium targets, 1903.08544.

[15] CLAS collaboration, S. Diehl, SIDIS Pion Beam Spin Asymmetries with CLAS 12 at 10.6 GeV, PoS DIS2019 (2019) 173.

[16] HERMES collaboration, G. Schnell, Longitudinal double-spin asymmetries in semi-inclusive deep-inelastic scattering of electrons and positrons by protons and deuterons, PoS DIS2019 (2019) 202. 
[17] HERMES collaboration, A. Airapetian et al., Longitudinal double-spin asymmetries in semi-inclusive deep-inelastic scattering of electrons and positrons by protons and deuterons, Phys. Rev. D99 (2019) 112001, [1810.07054].

[18] M. Anselmino, A. Efremov, A. Kotzinian and B. Parsamyan, Transverse momentum dependence of the quark helicity distributions and the Cahn effect in double-spin asymmetry A(LL) in Semi Inclusive DIS, Phys. Rev. D74 (2006) 074015, [hep-ph/ 0608048 ].

[19] S. Bastami, H. Avakian, A. V. Efremov, B. U. Musch, B. Parsamyan, A. Prokudin et al., Applications of the WW-type approximation to SIDIS, PoS DIS2019 (2019) 211, [1907.11165].

[20] S. Bastami et al., Semi-Inclusive Deep Inelastic Scattering in Wandzura-Wilczek-type approximation, JHEP 06 (2019) 007, [1807. 1060 6].

[21] F. Bradamante, V. Barone, A. Bressan, A. Kerbizi, A. Martin, A. Moretti et al., Future SIDIS measurements with a transversely polarised deuteron target at COMPASS, PoS DIS2019 (2019) 267.

[22] M. Radici, Update on phenomenological extraction of the proton tensor charge, PoS DIS2019 (2019) 199.

[23] COMPASS collaboration, A. Martin, Future SIDIS measurements with a transversely polarised deuteron target at COMPASS, PoS DIS2019 (2019) 267.

[24] V. Barone, F. Bradamante, A. Bressan, A. Kerbizi, A. Martin, A. Moretti et al., Transversity distributions from difference asymmetries in semi-inclusive DIS, Phys. Rev. D99 (2019) 114004 , [1902.08445].

[25] A. Martin, F. Bradamante and V. Barone, Extracting the transversity distributions from single-hadron and dihadron production, Phys. Rev. D91 (2015) 014034, [1412.5946].

[26] CLAS 12 collaboration, C. Dilks, Probing Dihadron Fragmentation Functions and Twist-3 Parton Distribution Functions at CLAS12, .

[27] COMPASS collaboration, S. Sirtl, Azimuthal asymmetries in SIDIS di-hadron muoproduction off longitudinally polarized protons at COMPASS, 1702.07317.

[28] W. Yang and Z. Lu, On the $\sin \phi_{R}$ single longitudinal spin asymmetry in dihadron production in SIDIS, PoS DIS2019 (2019) 215.

[29] COMPASS collaboration, B. Parsamyan, Transversely polarized Drell-Yan measurements at COMPASS, PoS DIS2019 (2019) 195, [1908.01727].

[30] COMPASS collaboration, M. Aghasyan et al., First measurement of transverse-spin-dependent azimuthal asymmetries in the Drell-Yan process, Phys. Rev. Lett. 119 (2017) 112002, [1704.00488].

[31] Y. Fu, S. Xue, X. Wang, Z. Lu and I. Schmidt, Sivers Asymmetry in pi N Drell-Yan process at COMPASS within TMD factorization, PoS DIS2019 (2019) 214.

[32] COMPASS collaboration, R. Longo, Measurement of $\mathbf{q}_{\mathbf{T}}$-weighted transverse-spin-dependent azimuthal asymmetries at COMPASS, PoS DIS2019 (2019) 186, [1908.03310].

[33] D. Boer, Investigating the origins of transverse spin asymmetries at RHIC, Phys. Rev. D60 (1999) 014012, [hep-ph/9902255].

[34] COMPASS collaboration, A. Vidon, Exclusive single-photon muoproduction at COMPASS, .

[35] CLAS 12 collaboration, F. Bossu, First data on Deeply Virtual Compton Scattering with CLAS12 at 10.6 GeV Electron Beam, . 
[36] COMPASS collaboration, R. Akhunzyanov et al., Transverse extension of partons in the proton probed in the sea-quark range by measuring the DVCS cross section, Phys. Lett. $\mathrm{B} 793$ (2019) 188-194.

[37] PHENIX collaboration, N. Lewis, Transverse Single-Spin Asymmetries of Midrapidity Eta Mesons at PHENIX , POS DIS2019 (2019) 185.

[38] PHENIX collaboration, V. Papavassiliou, Nuclear-dependence of transverse, single-spin asymmetries in charged hadron production in PHENIX, .

[39] PHENIX collaboration, C. Aidala et al., Nuclear-modification factor of charged hadrons at forward and backward rapidity in $p+A l$ and $p+A u$ collisions at $\sqrt{s_{N N}}=200 \mathrm{GeV}, 1906.09928$.

[40] STAR collaboration, N. Lukow, Constraining the Polarized Gluon Distribution Function of the Proton with Recent STAR Measurements, PoS DIS2019 (2019) 187.

[41] STAR collaboration, J. Adam et al., Longitudinal double-spin asymmetry for inclusive jet and dijet production in pp collisions at $\sqrt{s}=510 \hat{a} \breve{A} L^{\prime} \hat{a} \breve{A} L^{\prime} G e V$, Phys. Rev. D100 (2019) 052005, [1906.02740].

[42] STAR collaboration, A. Kraishan, Measurement of the Longitudinal Single Spin Asymmetries for $W$ Boson Production in Polarized Proton-Proton Collisions at $\sqrt{s}=510 \mathrm{GeV}$ at STAR, .

[43] STAR collaboration, J. Adam et al., Measurement of the longitudinal spin asymmetries for weak boson production in proton-proton collisions at $\sqrt{s}=510$ GeV, Phys. Rev. D99 (2019) 051102, [1812.04817].

[44] PHENIX collaboration, A. Adare et al., Cross section and longitudinal single-spin asymmetry $A_{L}$ for forward $W^{ \pm} \rightarrow \mu^{ \pm} v$ production in polarized $p+p$ collisions at $\sqrt{s}=510$ GeV, Phys. Rev. D98 (2018) 032007, [1804.04181].

[45] F. Hekhorn and M. Stratmann, NLO corrections to heavy flavour distributions in polarized deep-inelastic scattering, PoS DIS2019 (2019) 177.

[46] F. Hekhorn and M. Stratmann, Next-to-Leading Order QCD Corrections to Inclusive Heavy-Flavor Production in Polarized Deep-Inelastic Scattering, Phys. Rev. D98 (2018) 014018, [1805. 09026 ].

[47] T. Bhattacharya, V. Cirigliano, S. Cohen, R. Gupta, H.-W. Lin and B. Yoon, Axial, Scalar and Tensor Charges of the Nucleon from 2+1+1-flavor Lattice QCD, Phys. Rev. D94 (2016) 054508, [1606.07049].

[48] C. Alexandrou et al., Nucleon scalar and tensor charges using lattice QCD simulations at the physical value of the pion mass, Phys. Rev. D95 (2017) 114514, [1703.08788].

[49] H. Moutarde, P. Sznajder and J. Wagner, Border and skewness functions from a leading order fit to DVCS data, Eur. Phys. J. C78 (2018) 890, [1807 . 07620].

[50] S. Bhattacharya, C. Cocuzza and A. Metz, Studying twist-2 GPDs through quasi-distributions in a scalar diquark model, PoS DIS2019 (2019) 169.

[51] S. Bhattacharya, C. Cocuzza and A. Metz, Generalized quasi parton distributions in a diquark spectator model, Phys. Lett. B788 (2019) 453-463, [1808.01437].

[52] A. Amor-Quiroz, M. Burkardt and C. Lorce, The angular momentum decomposition in the scalar diquark model, PoS DIS2019 (2019) 168.

[53] S. Rodini, B. Pasquini and A. Bacchetta, Lensing function relation in Hadrons, PoS DIS2019 (2019) 200. 
[54] V. Bertone, I. Scimemi and A. Vladimirov, Extraction of unpolarized quark transverse momentum dependent parton distributions from Drell-Yan/Z-boson production, JHEP 06 (2019) 028, [1902.08474].

[55] I. Scimemi, A. Tarasov and A. Vladimirov, Collinear matching for Sivers function at next-to-leading order, JHEP 05 (2019) 125, [1901.04519].

[56] B. Wang, J. O. Gonzalez-Hernandez, T. C. Rogers and N. Sato, Large Transverse Momentum in Semi-Inclusive Deeply Inelastic Scattering Beyond Lowest Order, Phys. Rev. D99 (2019) 094029, [1903.01529].

[57] J. O. Gonzalez-Hernandez, T. C. Rogers, N. Sato and B. Wang, Challenges with Large Transverse Momentum in Semi-Inclusive Deeply Inelastic Scattering, Phys. Rev. D98 (2018) 114005, [1808.04396].

[58] A. Simonelli, M. Boglione and O. Gonzalez, The impact of the errors of collinear functions in describing unintegrated SIDIS data, PoS DIS2019 (2019) 168.

[59] A. Kerbizi and L. Lonnblad, Inclusion of the ${ }^{3} P_{0}$ model in PYTHIA 8, 1909.02280.

[60] A. Kerbizi, X. Artru, Z. Belghobsi, F. Bradamante and A. Martin, Recursive model for the fragmentation of polarized quarks, Phys. Rev. D97 (2018) 074010, [1802. 00962 ]. 